\title{
South African construction and demolition waste procedure and its sourced material effects on concrete
}

\author{
Johannes Bester", Deon Kruger, and Broadley Miller \\ University of Johannesburg, PO Box 524, Auckland Park, 2006, Johannesburg, South Africa
}

\begin{abstract}
This paper presents the results of a study into the use of fine aggregate from construction and demolition waste (C\&DW) in the South African construction industry, and the impact this has on the important fresh and hardened properties of concrete when used as partial replacement for virgin materials. The C\&DW were tested at replacement levels of $0 \%, 25 \%$, $50 \%, 75 \%$ and $100 \%$. The recycled fine aggregate was pre-soaked to mitigate the expected effect of higher water absorption of recycled concrete aggregate (RCA). However, pre-soaking of the fines attributed to the poor performance of the fresh and hardened properties of the concrete. The presoaking procedure used was problematic when used with fine aggregates and caused a large amount of excess water to be added to the concrete mix as a result. This study highlights the importance of planning the demolition process of a structure to ensure that the best possible quality of RCA can be extracted. The study revealed that the use of recycled fine aggregate as a replacement of virgin materials in the manufacture of concrete has a much greater negative impact than the use of coarse recycled aggregates. In addition, the pre-soaking procedure of fine aggregates warrants special attention.
\end{abstract}

\section{Introduction}

For many years and across the globe, the use of natural resources and the abuse of the environment have been seen as necessary "costs of development", but recently the drive for a greener, more sustainable construction industry has been on the rise worldwide. The introduction of green building rating with systems such as those employed by Leadership in Energy and Environmental Design (LEED) in the United States of America and the Green Star rating system in South Africa (SA) have changed the global view of green technology. This change in public perception has led to the demand, and subsequently, the value of including of "green" properties in materials has being on the rise.

This international trend has resulted in a rethink of the construction industry as a whole worldwide, resulting in what is known as sustainable development, which the Bruntland Report defines as: "Development that meets the needs of the present, without compromising

\footnotetext{
*Corresponding author: jannesb@uj.ac.za
} 
on the ability of the future generations to meet their needs." According to the triple bottom line theorem of Sustainable Development, Sustainable Development comprises of three main parts, namely: Economic-, Environment- and Social factors [1]. All three of these factors need to be considered and balanced in order to make a project or product as sustainable as possible and thus meet the "Triple Bottom Line" of sustainable development.

\section{The construction and demolition waste industry}

The idea of recycling of construction and demolition waste (C\&DW) is not a new one, but one that many developed countries such as those in the European Union and Japan have implemented very successfully for a number of years now. Various studies have shown that the C\&DW waste streams in Europe alone in the late 90's stood at around 200-330 million tons per year. This type of waste was generally made-up of around $67 \%$ of masonry and old concrete rubble and it is assumed that the waste make-up is the same in developed countries worldwide. Recycling of this C\&DW generated across Europe however stood at only $28 \%$, whereas some countries such as the Netherlands recycle up to $95 \%$ of their construction and demolition waste [2]. Various international regulatory bodies the world over has noticed this possibility for growth of C\&DW recycling. The EU even has short term goals in place to enforce the recycling of between $50-90 \%$ of all the construction and demolition waste generated in their jurisdiction [3].

The C\&DW stream in South Africa (SA) alone is estimated at around 5-8 million tons [4]. This shows that there is a massive opportunity for growth in the recycling industry worldwide as well as in SA with regards to the recycling of C\&DW. SA still has vast open spaces and natural aggregate resources are still available, therefore it is not surprising that SA is lagging behind in terms of its development of measures to promote the recycling of C\&DW. However, as the available area for landfill sites are reduced, natural resources that are depleting and the pressures from global markets increase, such as with the need for ISO 9000 and ISO 14000 requiring more companies to have quality environmental management systems, SA might soon see the need for effective recycling of C\&DW [5].

\section{Aspects of construction and demolition waste recycling}

To truly partake in the sustainable development process one has to look at all the aspects of waste minimisation throughout the project life-cycle. The $4 \mathrm{R}$ 's of waste minimisation are [6]:

1. Recovery

2. Reuse

3. Recycling

4. Reduction

The recycling of construction and demolition waste could have a number of positive aspects, such as:

1. Reduced impact on the environment, through lowering the demand for virgin materials;

2. Reduced cost of materials, as recycled materials are usually less expensive than virgin materials;

3. Reduced cost of transportation of material, as demolition waste could be used on the same site or in a nearby area;

4. Reduced impact on the environment, through a decrease in the need to landfill the waste, the lifespan of landfills could be increased;

5. Reduced landfilling costs. 
At present the major uses of recycled construction and demolition waste has been for roadfills and backfills [3]. This unfortunately is considered more of a "down-cycling" process resulting from the perception that recycled aggregates are of inferior quality and thus virgin materials are still used for the majority of new concrete production. This is because past studies by institutions such as RILEM (The International Union of Testing and Research Laboratories for Materials and Structures), ACI (American Concrete Society) and Building Contractors Society of Japan (B.C.S.J) have shown a large degree of variability in the quality of the recycled material and the concrete made therewith.

Tests on the effects of recycled aggregates when used in the production of new concrete have been conducted internationally for over 30 years and from this some properties and effects have been proven to be quite common. These general findings have shown that concrete mixes using recycled concrete aggregate (RCA) can be designed in much the same way as those using natural aggregates, provided that the higher water absorption in RCA is appropriately accounted for when determining the water content.

In an effort to try and minimise the great variation in the fresh properties and strength results obtained when using recycled aggregates in the production of new concretes, most countries that allow the use of recycled aggregate in structural concrete have adopted necessary criteria or classification standards that the recycled aggregate must adhere to in order to be deemed safe for the use in the production of new concrete. These classification measures have seemed to be quite effective in minimising the risk when recycled aggregates are used, as well as giving the users a guide to the effects that the aggregates would have on the concrete as well as ways in which to mitigate them.

\section{Experimental design}

The RCA that was used in this research was sourced from the demolition of an unknown structure. As the case generally would be in South Africa, the RCA that was produced had little control over the recycling process, thus it contained various contaminants (Figure 1). After crushing and sieving the material in the laboratory, it was found to contain mostly fine particles and hence the subsequent tests will be on the use of the waste material for the replacement of the fines part of the concrete mix as described by the Fulton's Concrete Technology handbook [7].

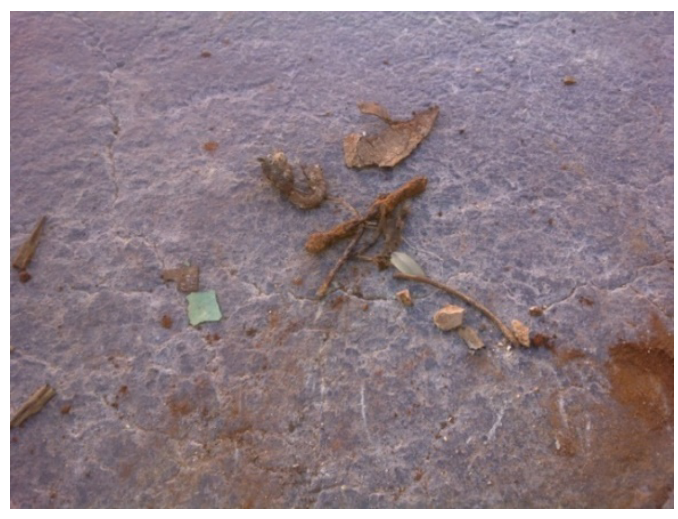

Fig. 1. Contaminants in the RCA.

A control mix (Table 1), simulating a ready-mix concrete, was made by the Afrisam Roodepoort Plant. Replacement levels of $25 \%, 50 \%, 75 \%$ and $100 \%$ by mass was used to determine how the level of replacement affected the fresh and hardened properties of the concrete. 
Table 1. Control concrete mix design.

\begin{tabular}{|c|c|}
\hline Description & Control mix \\
\hline w:c & 0.67 \\
\hline Water (L) & 195 \\
\hline CEM II A-M (S-V) 42.5R (kg) & 290 \\
\hline Eikenhof crusher sand (kg) & 990 \\
\hline 22mm Eikenhof stone (kg) & 1075 \\
\hline Slump (mm) & 60 \\
\hline
\end{tabular}

The $25 \%, 50 \%, 75 \%$ and $100 \%$ replacement mixes were made in accordance with the procedure set out in SANS 5861-1: Concrete tests - Mixing fresh concrete in the lab. After casting of the moulds, the test cubes was cured in water in accordance with the standards set out in SANS 5861-3: Concrete tests - Making and curing of test specimens. A total of 15 test cubes and 3 test beams of each of the different mixes were manufactured. It must be mentioned at this point in time that no economic comparisons are considered for this project.

\subsection{Fresh concrete properties}

The tests used to evaluate the effect of the recycled fine aggregate on the fresh properties of the concrete compared to that of the control mix were the slump test (SANS 5862-1: 2006), slump retention and total bleed test (ASTM C232/C232M) and the setting time test (SANS 501 963-3: 2006).

\subsection{Hardened concrete properties}

In order to generate a strength gain curve 15 cubes sized $100 \mathrm{~mm} \times 100 \mathrm{~mm} \times 100 \mathrm{~mm}$ were crushed at 1, 3, 7 and 28 days from the day of casting, in accordance with SANS 5863: Concrete tests - Compressive strength of hardened concrete. The flexural strength was determined on 28 days, in accordance with SANS 5863: Concrete tests - Flexural strength of hardened concrete. These specimen sizes were $500 \mathrm{~mm} \times 100 \mathrm{~mm} \times 100 \mathrm{~mm}$, and there were 15 specimens tested. A single load was applied at mid-span.

\subsection{Pre-soaking}

The aggregates were pre-soaked in an attempt to mitigate most of the negative effects caused by recycled aggregates when used in concretes. The pre-soaking procedure consisted of submerging the material in water an hour before being used. After 40 minutes the material was removed from the water and allowed to air dry for 20 minutes. This was done to allow excess water to drain off and evaporate, in order to have the material as close as possible to a saturated surface dry condition.

Pre-soaking on the aggregates could also have a negative effect on the results. The very fine dust particles could be washed away during draining after the pre-soaking, which could have an effect the bleeding characteristics. In addition, if the material washed away consisted 
of un-hydrated cement particles as expected, it could also have an effect on the compressive and flexural strengths achieved.

\section{Results}

One of the material classification tests that was performed on the sand, was the relative density (RD) test. The RD of the sand used in the control mix was 2.89 , whereas the RD of the RCA sand was 2.57 . The lower RD could be attributed to the usually more porous nature of RCA.

Figure 2 shows the gradings of the sand used in the control mix and the demolition waste sand (DW) used to manufacture the RCA. It also indicates the envelope used in the concrete mix design. The results indicates that the RCA fines are very fine, and falls mostly outside the fines limit, as set out by The Concrete Institute [2]. A possible reason for this is the amount of masonry present in the RCA.

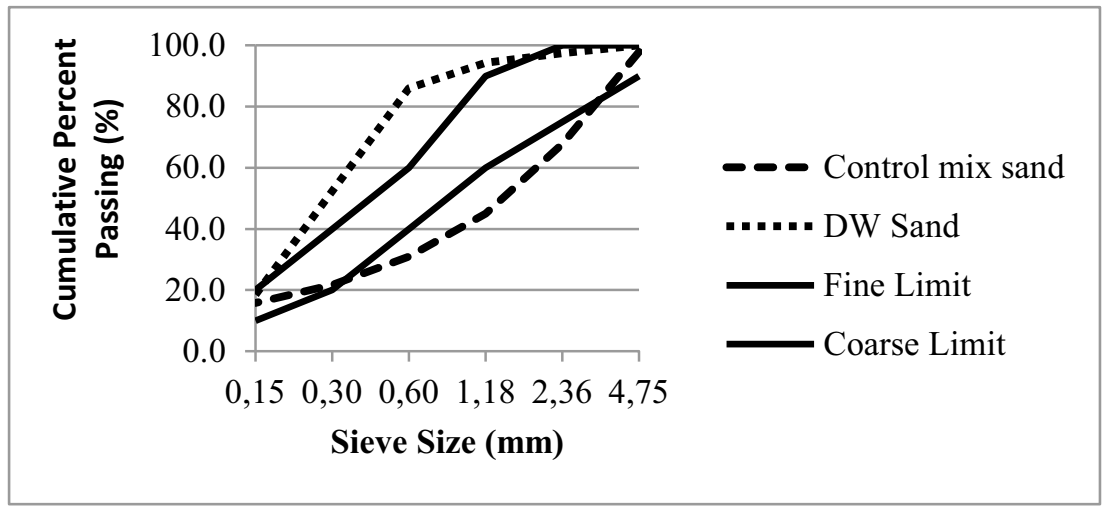

Fig 2. Grading envelope, control sand and DW sand gradings.

\subsection{Fresh properties results}

\subsubsection{Slump}

Figure 3 indicates that here is a massive increase in the slump value of the mixes utilizing the DW aggregate as compared to the control mix. The RCA mixes had a small positive increasing trend in the slump value as the percentage DW replacement was increased. Unfortunately, due to time constraints, the material was not left to air dry completely to the saturated surface dry condition desired. The effect of the increased capillary action of the finer material that would make it difficult for excess water to drain off after the pre-soaking, the material was not in a proper saturated surface dry condition and most likely had a large degree of surface water present. This surface water is exacerbated by the material's small particle size, which means that the percentage water retained by the recycled material is much higher for the fine material than it would have been for a larger particle size. All the mixes however still possessed good cohesion. 


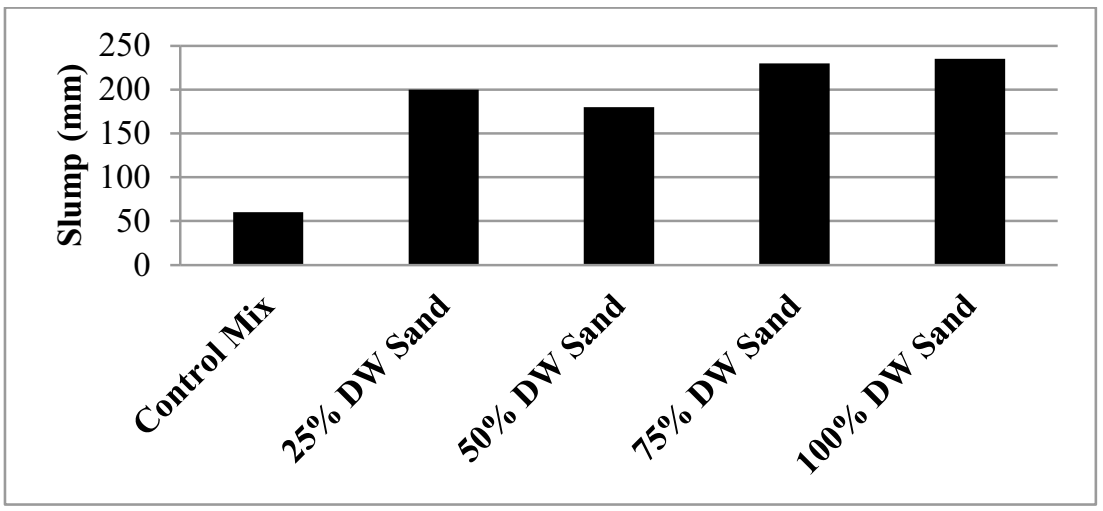

Fig. 3. Slump results.

\subsubsection{Slump retention}

When the trend lines produced by the slump retention results of the different mixes containing the demolition waste is compared with that of the control mix (Figure 4) there is a slight trend of reduced slump retention or faster setting rate than expected. However the trend does not increase as the level of replacement increases. The reason for this reduced slump retention could be as a result of the higher water absorption of the demolition waste material, thus effectively taking water from the mix. Thus, the pre-soaking procedure used for this material may have been inadequate and a longer pre-soak time could have been necessary.

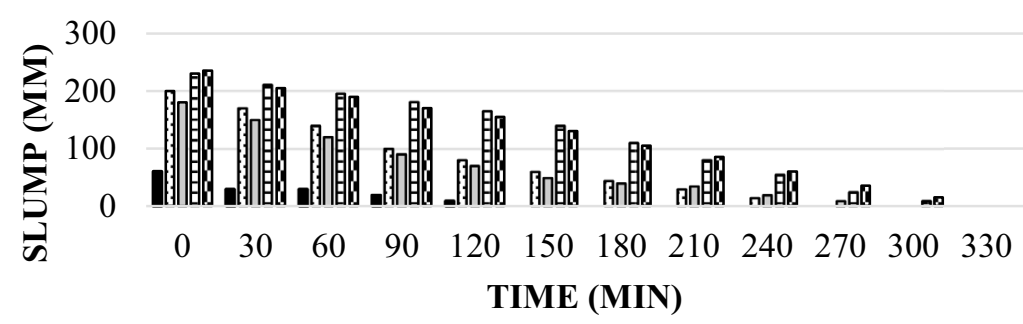

-Control Mix $\square 25 \%$ DW Sand $\quad \square 50 \%$ DW Sand

$\square 75 \%$ DW Sand $\square 100 \%$ DW Sand

Fig. 4. Slump retention.

\subsubsection{Setting time}

The setting and hardening process of Portland cement can be divided into 3 distinct stages [8], these stages are:

1. Dormant Stage - paste remains plastic and workable

2. Setting stage - the paste is stiff and unworkable

3. Hardening stage - the paste is a rigid solid and gains strength with time 


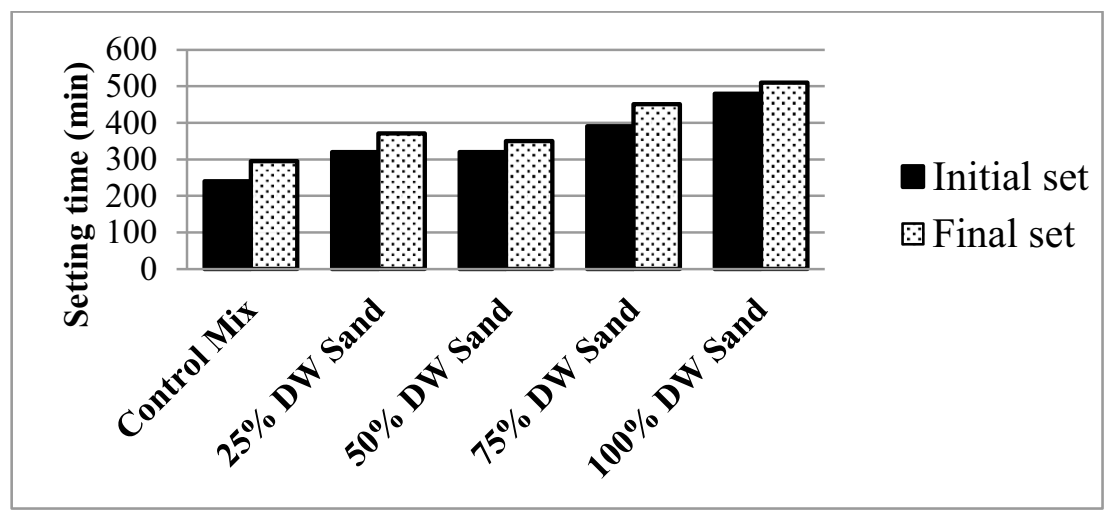

Fig. 5. Setting time.

\subsubsection{Bleed volume}

The transition between the dormant and setting stage is known as the initial set and the transition between the setting and hardening stages is known as the final set. As can be seen from the Figure 5, there is an almost linear increase in the setting times achieved for the mixes containing the demolition waste sand material compared with that of the control mix. It can therefore be deduced that an increase in the amount of recycled fines would result in an increase in the setting time of the mix. The high workability in conjunction with the increased setting times of the mix could be an indicator of a high degree of free water in the mix. If the setting stage of the $50 \%$ replacement is taken as an outlier, then the results in Figure 5 would suggest that the setting stages of the mixes containing the demolition waste material reduces as the level of replacement increases.

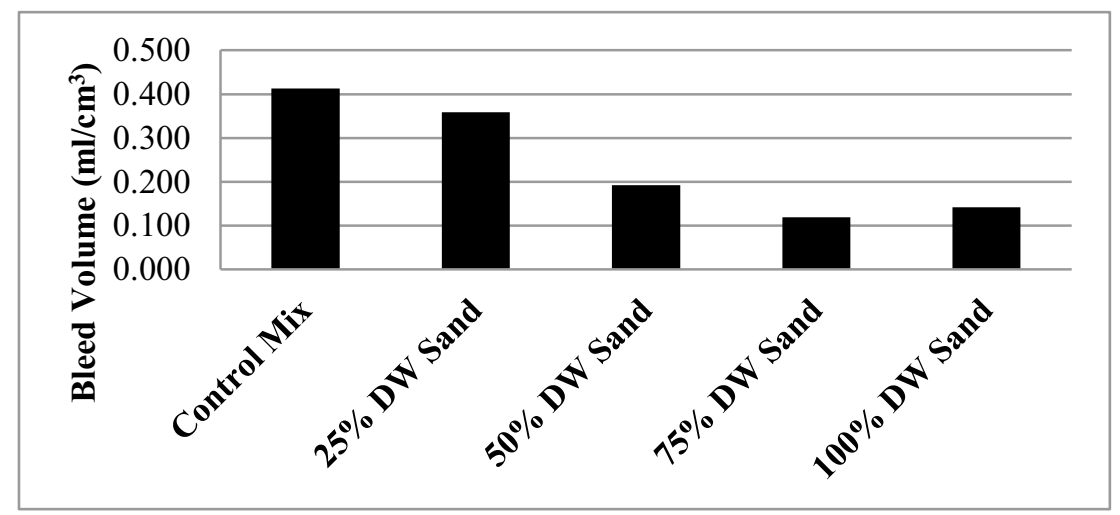

Fig. 6. Bleed volume.

It is important to note that the bleed volume results in this study were only the bleed volumes measured on the surface of the concrete after the 280 minute test period, and not the total bleed volume of the concrete. This is because weight measurements of the test samples after being oven-dried were not measured and therefore, the total bleed volumes could not be calculated. The bleeding of concrete is affected by 2 main factors, the relative density of the aggregates in the concrete mix as well as the amount extremely fine material present in the concrete mix. As can be seen in Figure 6, the bleed volumes of the concretes containing the demolition waste material showed lower bleeding than that of the reference mix, and the results also showed a trend of decreasing bleed volumes as the level of replacement increased. 
This was unexpected as the slumps earlier pointed to excess water present in the concrete mixes and the grading of the material, in Figure 1, also showed a reduced amount of very fine or dust particles present, as well as the assumption that much of the finer particles would have been washed away during the pre-soaking procedure. All of these factors should have increased the bleed volumes experienced. However, the lower relative density of the recycled aggregate as well as the higher water absorption of the demolition waste aggregate seems to have been sufficient to surpass all of these factors and thus effectively reduced the amount of bleeding of the concrete mixes containing the demolition waste sand material.

\subsection{Hardened properties results}

\subsubsection{Compressive strength development}

It can be seen from Figure 7 that as the replacement of the virgin aggregate with that of recycled demolition fines increases, there is a drastic reduction in the compressive strength development of the concrete. This progressive loss in compressive strength could be attributed to the gradual increase in the lower quality demolition waste sand. This effect could have been exacerbated by the high volume of pores in the DW aggregate present in the concrete mixes leading to an increase in the voids of the concrete and thus a loss in the concrete's strength. The mixture, as it stands, is not really advisable for any use, due to the severely reduced strength for the amount of cement added, effectively making the concrete uneconomical to use.

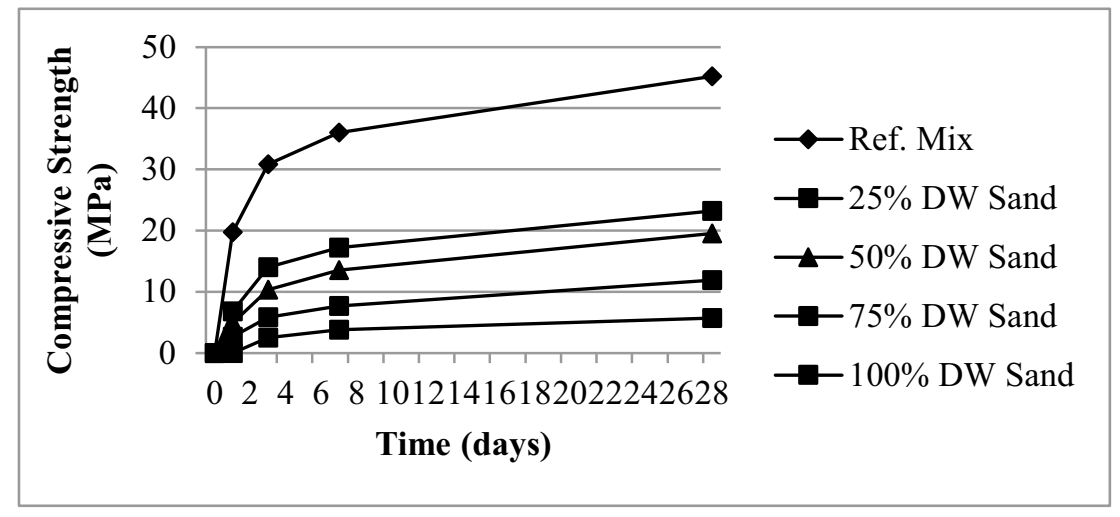

Fig. 7. Compressive strength development curves.

\subsubsection{Flexural strength}

It can be seen from Figure 8 that the flexural strength of the concrete decreased with the increase in sand replacement. It is said that concrete mixes made with virgin aggregates typically have a compressive strength to flexural strength ratio of around 1:10, and the control mix stays true to this assumption. The recycled demolition waste sand showed ratios ranging between 1.33:10 to 2.4:10. The ratio increase with an increase in the percentage replacement. This could be as a result of the better interfacial bond strength of the material to the new cement mortar because of its rougher texture caused by the attached cement mortar paste. 


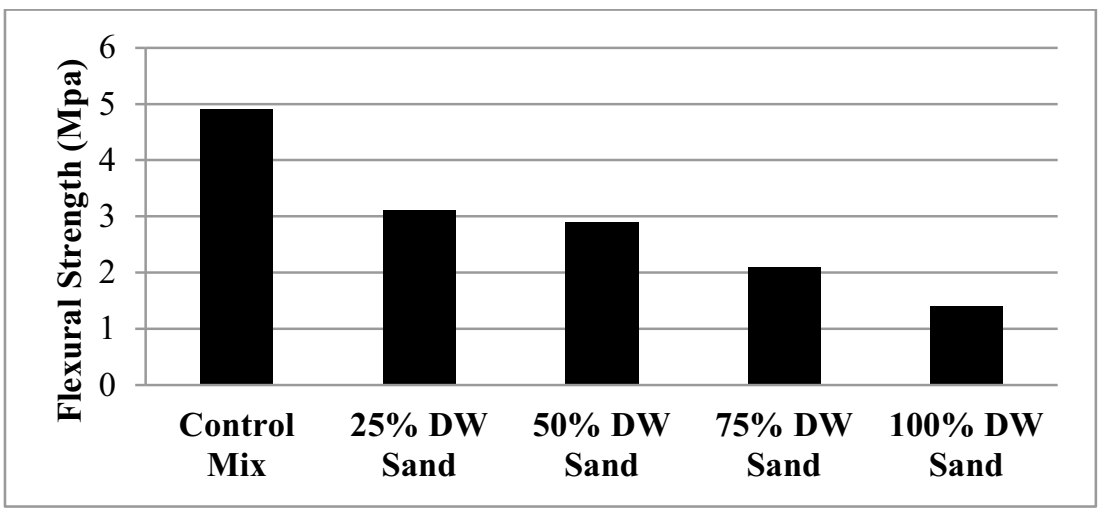

Fig, 8. Flexural strength.

\section{Conclusion}

The masonry contaminants found in the C\&DW material caused the concrete to react in a different manner to that which is usually expected from a clean recycled aggregate concrete. The fresh properties of the concrete showed a marked increase in the slump, and a large drop in the slump retention. The initial set, and final set increased as the replacement of natural aggregate increased. The bleed volume also showed a marked decrease. There was a marked decrease in the compressive strength development of the concrete, and the flexural strength also decreased. There was a marked increase in the ratio of concrete flexural strength to compressive strength.

It can be concluded that to use of recycled aggregate from the demolition of an unknown structure, which has little control over the recycling process will negatively affect the properties, as well as the durability. To address these negative effects, adjustments needs to be made to the mix design, thus increasing the cost of the concrete.

\section{References}

1. G. Owens (Editor), Fundamentals of Concrete, The Concrete Institute, Midrand (2013)

2. P. K. Mehta, Reducing the environmental impact of concrete. Concrete International. (2007)

3. E. K. Lauritzen, Recycling Concrete - An Overview of Challenges and Opportunities. In Liu, T.C. \& Meyer, C., eds. Recycling Concrete and Other Materials for Sustainable Development., ACI International - SP - 219. (2005)

4. K. Benjamin, Performance of concrete made with commercially produced recycled coarse and fine aggregate in Cape Peninsula. Master's Thesis. UCT. (2004)

5. G. Grieve, Aggregates for Concrete. Chapter 3 in Fulton's concrete technology. Midrand: The Cement \& Concrete Institute, pp.55-56. (2009)

6. P.A. Vesilind, W. Worrell, D. Reinhart, Solid waste engineering. California. Thomson Learning. (2002)

7. B. Addis, J. Goodman, Concrete mix design. Chapter 11 in Fulton's concrete technology. Midrand: The Cement \& Concrete Institute, pp. 219-224. (2009)

8. J. Kellerman, S. Crosswell, Properties of fresh concrete. Chapter 6 in Fulton's concrete technology. Midrand: The Cement \& Concrete Institute, pp. 83-94. (2009) 